\title{
Digital Literacy: A Barrier to Adoption of Connected Health Technologies in Older Adults
}

\author{
Lisa Gualtieri ${ }^{1}$, PhD, ScM; Jeffrey Phillips ${ }^{2}$, MD; Sandra Rosenbluth ${ }^{1}$, MS; Steph Synoracki ${ }^{1}$
}

${ }^{1}$ Department of Public Health and Community Medicine, School of Medicine, Tufts University, Boston, MA, United States

${ }^{2}$ Wellesley Family Care Associates, Wellesley, MA, United States

\author{
Corresponding Author: \\ Lisa Gualtieri, $\mathrm{PhD}$, ScM \\ Department of Public Health and Community Medicine \\ School of Medicine \\ Tufts University \\ 136 Harrison Avenue \\ Boston, MA, \\ United States \\ Phone: 16176360438 \\ Email: lisa.gualtieri@tufts.edu
}

\section{Abstract}

Background: Adoption and effective use of digital health technologies is especially important for older adults, who bear the greatest burden of chronic disease. However, some older adults lack the digital literacy skills needed to reap the benefits of these tools. Despite the greater infiltration of smartphones in all age groups, including older adults, smartphone ownership does not equate to adept smartphone use. Impediments to smartphone use can impede adoption and use of digital health technologies that rely on apps.

Objective: Our objectives were to 1) determine the barriers to adoption and use of digital health technologies, specifically wearable activity trackers, by older adults; and 2) identify and test facilitators to adoption and use.

Methods: We recruited more than 100 older adults aged 50-75 who owned a smartphone for our tracker studies. We provided each participant with a tracker and hands-on support for its setup and use. Support was tailored to each participant's level of comfort and adeptness with his or her smartphone and new tracker, with the goal of having participants leave with a working tracker and an app they could engage with.

Results: While all participants owned a smartphone, we identified several barriers to successful engagement with their own smartphones as well as with their new trackers. Some older model or pay-as-you-go phones could not download apps. Many participants required assistance to download and setup an app, as well as sync their tracker to the app. In several cases, the use of jargon such as "sync with Bluetooth" required an explanation. Some app features required guidance, such as changing the default of 10,000 steps to one that was more achievable and realistic for older adults. Furthermore, the design of some tracker faces and bands were preferred or more comfortable for participants. We found that once participants were able to overcome barriers to setup through hands-on training, other facilitators, such as a visual reminder of their commitment to physical activity and the immediate gratification of seeing their activity reflected in the tracker and the app, kept them engaged with the tracker.

Conclusions: The digital health market is growing rapidly and has great potential to improve health outcomes, yet it is failing to reach and meet the needs of older adults. Poor digital literacy skills impede adoption of digital health technologies, and their effective use once adopted. While a high-touch approach to helping older adults overcome barriers to adoption and use of digital health technologies has proven to be a successful facilitator in our studies, the challenge is in bringing this approach to scale and applying it to both consumer- and medical-grade technologies. In future studies, we hope to build on this knowledge and further identify design and training solutions that can be scaled to increase successful engagement of digital health technologies by older adults.

(iproc 2018;4(2):e11803) doi: $\underline{10.2196 / 11803}$

\section{KEYWORDS}

barriers; digital literacy; older adults; smartphone; trackers 
Edited by T Hale; this is a non-peer-reviewed article. Submitted 02.08.18; accepted 30.08.18; published 17.09.18.

Please cite as:

Gualtieri L, Phillips J, Rosenbluth S, Synoracki S

Digital Literacy: A Barrier to Adoption of Connected Health Technologies in Older Adults iproc 2018;4(2):e11803

URL: http://www.iproc.org/2018/2/e11803/

doi: $\underline{10.2196 / 11803}$

PMID:

CLisa Gualtieri, Jeffrey Phillips, Sandra Rosenbluth, Steph Synoracki. Originally published in Iproceedings (http://www.iproc.org), 17.09.2018. This is an open-access article distributed under the terms of the Creative Commons Attribution License (https://creativecommons.org/licenses/by/4.0/), which permits unrestricted use, distribution, and reproduction in any medium, provided the original work, first published in Iproceedings, is properly cited. The complete bibliographic information, a link to the original publication on http://www.iproc.org/, as well as this copyright and license information must be included. 